\title{
Sinusitis de origen odontogénico.
}

\section{Acute odontogenic sinusitis.}

Kimberly Arias Amador Sofia Rojas Díaz² Viviana Saenz Ramirez ${ }^{3}$

1, y 2 Licenciado en medicina y cirugía. Caja costarricense seguro social, Cartago Costa Rica.

3 Licenciado en medicina y cirugía. Caja costarricense seguro social, Heredia Costa Rica.

Contacto: kimariasamador@hotmail.com rojasdiazso@hotmail.com vivi.saenzr93@gmail.com

\section{RESUMEN}

Sinusitis es la inflamación de uno o más senos paranasales, puede ser aguda, crónica o recurrente. (1)

Dicha inflamación puede ser consecuencia de la invasión por bacterias, virus u hongos, puede ser causada por la evolución de una lesión en el diente con formación de pus periapical que avanza en el interior de la cavidad sinusal. (2)

La evaluación temprana de los pacientes que manifiesten invasiones dentales es esencial con el fin de evitar las complicaciones clínicas que puedan detonar en la penetración de dicha cavidad. Del mismo modo, se debe estar atento a realizar un diagnóstico temprano y la respectiva cobertura antibiótica en conjunto con las pruebas de sensibilidad antibiótica en pacientes con empeoramiento del cuadro clínico de origen odontogénico.

Palabras Clave: Pansinusitis, agudo, odontogénico.

\section{ABSTRACT}

\section{Cómo citar:}

Arias Amador, K., Rojas Díaz, S. ., \& Saenz Ramirez, V. . Pansinusitis de origen odontogénico. Revista Ciencia Y Salud Integrando Conocimientos, 5(4), Pág. 23-27. https:// doi.org/10.34192/

cienciaysalud.v5i4.287

Sinusitis is defined as the inflammation of one or more paranasal sinuses, that can be classified in acute, chronic or recurrent. (1)

This inflammation could occur as a result of invasion of bacteria, viruses or fungi, which can be caused by the evolution of a lesion in the tooth with formation of periapical pus that advances into the sinus cavity. (2)

Early evaluation of patients who manifest dental invasions is essential in order to avoid clinical complications that may trigger the penetration of the cavity. In the same way, care must be taken to make an early diagnosis and the respective antibiotic coverage in conjunction with antibiotic sensitivity tests in patients with worsening of the clinical manifestations of odontogenic origin.

Keywords: Pansinusitis, acute, odontogenic.

Recibido: 23/mar/2021

Aceptado: 25/ago/2021

Publicado: 04/sep/2021

\section{cc)(1)(2)(2)




\section{INTRODUCCIÓN}

En las infecciones causadas por bacterias que invaden los senos paranasales, el origen dental cumple un rol importante, generando una enfermedad grave debido a los potenciales daños a la zona orbitaria e intracraneal.

Las complicaciones ordinarias e intracraneales no son frecuentes en estas sinusitis, pero sí lo son en caso de compromiso etmoidal y frontal, las cuales no son de origen odontogénico. Las enfermedades periodontales tales como caries, abscesos, cuerpos extraños intraantrales y las perforaciones sinusales durante la exodoncia constituyen los focos de entrada para el desarrollo de este tipo de invasiones, una evaluación que involucre un diagnóstico a través de un examen físico exhaustivo e imágenes apropiadas constituye un método eficaz para la prevención de esta invasión. (3)

Esta relación anatomo patológica entre la sinusitis y los procesos infecciosos dentales se debe a que las raíces de los premolares y molares se encuentran inmediatamente por debajo del piso del seno maxilar y es de esta forma como existe evidencia que entre 10\% y 12\% de las infecciones del seno maxilar son infecciones odontógenas (4).

\section{MÉTODO}

Se utilizaron diferentes bases de datos de revistas médicas, en el idioma inglés y español, como Scielo, UpToDate, Medline y Pubmed. Los métodos de búsqueda se obtuvieron a través de la Biblioteca Nacional de Salud y el Sistema de Bibliotecas, documentación e información de la Universidad de Costa Rica (SIBDI) y bibliotecas virtuales.

Los criterios de inclusión se basaron en los resultados de las búsquedas en las diferentes bases de datos: "Sinusitis de origen odontogénica", "Infección de los senos paranasales"; se revisaron los artículos y se escogieron los que contenían la información necesaria para completar los objetivos de la revisión.

\section{EPIDEMIOLOGÍA}

Hay evidencia que desde el año 1943 Bauer describió la extensión de procesos inflamatorios periapicales hacia el seno maxilar. (5)

La sinusitis aguda de origen odontogénica es una afectación frecuente y bien establecida, la relación anatómica de continuidad de las piezas dentales con los espacios sinusales hace que se considere una causa frecuente de sepsis, incluso algunos estudios recientes reportan un $25 \%$ de los pacientes con diagnóstico de sinusitis maxilar tratados con cirugía se encontró una fuente dental de infección. (5)

La sinusitis odontogénica se reporta con mayor frecuencia en la cuarta década de la vida, siendo más prevalente en mujeres. (2)

\section{PATOGENIA}

Existe un flujo constante de microorganismos provenientes de la microbiota oral, que en ocasiones, son los causantes de la infección del tejido periapical, esto a su vez genera una respuesta inmune que permite la diseminación de la infección a los espacios aéreos de la región maxilo facial.

El aumento de la inflamación de los senos maxilares puede producir una reducción del oxígeno, disminución del flujo sanguíneo en la mucosa y depresión de la acción ciliar, este cambio que se ve reflejado en la disminución del pH de la zona infectada respalda el crecimiento de los microorganismos anaeróbios estrictos al proporcionar un potencial óptimo de óxido-reducción. (6)

Las causas más comunes de sinusitis odontogénica incluyen abscesos dentales y enfermedad periodontal que han perforado la membrana Schneidarian, irritación e infección secundaria causada por cuerpos extraños intraantrales y perforaciones sinusales durante la extracción del diente. $(5,6)$ 


\section{CIENCIA\&SALUD}

La iatrogenia $(55,97 \%)$ es la primera causa, seguida por periodontitis $(40,38 \%)$ y quistes odontógenos $(6,66 \%) .(2)$

El origen es de tipo polimicrobiano por lo que su tratamiento incluye uso de antimicrobianos contra los patógenos mixtos de la microbiota oral.

Los principales microorganismos que se encuentran en esta patología son las bacterias aerobias y anaerobias, con predominio de las anaerobias, Peptostreptococcus ssp., Fusobacterium spp., Prevotella spp. y Porphyromonas spp. (7)

\section{PRESENTACIÓN CLÍNICA}

Los casos de sinusitis maxilar odontogénica se manifiestan cuando la membrana schneideriana se lesiona o perfora, debido a una infección dental, implantes, piezas supernumerarias, traumatismo maxilar, cuerpos extraños, enfermedad de hueso maxilar, granuloma periapical o cirugía dental (extracción u osteotomías ortognáticas).

Se sospecha el origen dental cuando la aparición de la sinusitis acontece después de un episodio de odontalgia o procedimiento dental. El manejo multidisciplinario permite un adecuado tratamiento farmacológico y rehabilitador, de lo contrario, los cuadros pueden evolucionar a complicaciones graves con afectación encefálica y

orbitaria. Las complicaciones cerebrales por sinusitis presentan baja incidencia; sin embargo, cuando ocurren son de extrema gravedad, pues la propagación de la infección se asocia a mortalidad del paciente.

\section{TRATAMIENTO}

La literatura describe principios para el tratamiento de infecciones odontogénicas, los investigadores Velasco y Soto adaptaron el protocolo de Peterson y col. resumiéndolos en: (i) determinar la etiología y severidad de la infección, (ii) evaluar antecedentes médicos y sistema inmune del paciente, (iii) decidir lugar de atención, (iv) tratamiento quirúrgico, (v) soporte médico y nutricional, (vi) elección y prescripción de antibioterapia adecuada y (vii) evaluación periódica del paciente. (2)

En el tratamiento de la sinusitis odontogénica es necesario actuar sobre la causa del diente y la enfermedad en sí misma. Solo entonces es posible eliminar la infección existente y prevenir las recurrencias o complicaciones.

La combinación de la terapéutica farmacológica y quirúrgica, por lo general es necesaria para el tratamiento de la sinusitis odontogénica.

El tratamiento no traumático preconizado para la sinusitis odontogénica es con antibióticos de amplio espectro durante 3-4 semanas. Predominantemente las sinusitis frontales sin tratar o mal tratadas pueden progresar a una variedad de complicaciones, tales como: celulitis orbitaria, trombosis del seno cavernoso, meningitis, osteomielitis, absceso intracraneal y muerte. (8)

La penicilina se considera el fármaco de elección para el tratamiento de la sinusitis debido a su amplio espectro. En nuestro medio, el uso de amoxicilina es la penicilina sugerida para esta patología durante 7 a 14 días, siendo la pauta más aconsejada por 10 días, sin embargo, el desarrollo de bacterias resistentes limita su uso clínico. (9)

El paciente alérgico a este medicamento puede ser tratado con clindamicina o trimetoprimsulfametoxazol. Ante resistencia a la penicilina de los microorganismos en pacientes con sinusitis maxilar de origen odontogénico requiere la administración de agentes antimicrobianos eficaces contra estas bacterias. Esto incluye la clindamicina, cefalosporinas de segunda generación o carbapenem, e incluso una combinación de penicilina y un inhibidor de beta-lactamasa. El metronidazol se puede administrar si se asocia con un agente eficaz contra los estreptococos facultativos y aerobios. (10) 
Luego del tratamiento farmacológico es necesario el tratamiento definitivo del foco. El cirujano dentista juega un rol importante en la rehabilitación del diente, por lo que, ante un conducto infectado, se sugiere una endodoncia en los dientes afectados, posterior restauración y prótesis fija unitaria, realizando controles odontológicos periódicos para evitar sobreinfección.

En ausencia de mejoría del cuadro clínico, se sugiere la referencia del paciente para valoración por un otorrinolaringólogo, quien podrá realizar una cirugía endoscópica, con la finalidad de lograr un drenaje del seno infectado, preservando la mucosa la cual recuperará su función una vez resuelta la infección del seno. $(7,11)$

\section{DIAGNÓSTICO}

El Consenso Europeo sobre Rinosinusitis y Pólipos Nasales del año 2020, (EPOS 2020) establece la clasificación de las sinusitis, dividiendo las anteriores en primarias y secundarias, las cuales a su vez se subdividen en localizadas (unilaterales) y difusas (bilaterales), las sinusitis cuyo agente causal es a través de procesos odontogénicos, estaría categorizada según esta entidad como secundaria difusa. (12)

Para un diagnóstico certero, la evaluación dental y clínica exhaustiva es de suma importancia, ya que en ocasiones es difícil diferenciar los síntomas de origen dental de los sinusales; por ello es importante realizar una exploración física detallada; palpación de la pared anterior del maxilar, valorar edema de tejidos blandos en el vestíbulo incluso endoscopia nasal, aspiración y toma de cultivos, además de solicitar los estudios radiológicos adecuados, tomografías computarizadas de alta resolución y la tomografía computada volumétrica con haz cónico. $(7,13)$

La tomografía computada es el estudio de imagen estándar para valorar el seno maxilar, los cortes coronales, sagitales y axiales muestran la relación de abscesos odontogénicos periapicales y cuerpos extraños con el piso del seno. Además, permite el reconocimiento preciso de micosis sinusales, mostrando las condensaciones y calcificaciones de la bola fúngica. $(6,14)$

\section{CONCLUSIONES}

La pansinusitis de origen odontogénica representa un porcentaje nada despreciable, por lo que, debe ser correctamente diagnosticada por un equipo multidisciplinario y apoyada con exámenes complementarios. El tratamiento farmacológico de elección es antibióticos por vía parenteral, respaldado con hemocultivo y antibiograma, y analgesia durante la hospitalización. La monitorización de los signos y síntomas es clave para evaluar la fase de recuperación sistémica. La eliminación de la causa con endodoncia, rehabilitación dental y controles odontológicos subsiguientes, disminuye el riesgo de complicaciones futuras. (15)

\section{REFERENCIAS BIBLIOGRÁFICAS}

1. Mancilla Canelas Gonzalo, Mendoza Amatller Alfredo. Sinusitis. Rev. bol. ped. [Internet]. 2002 Jun [citado 2021 Mayo 27] ; 41( 2 ): 111-114. Disponible en: http://www.scielo.org.bo/scielo. php?script=sci_arttext\&pid=S1024-06752002000200014\&Ing=es.

2. Pansinusitis aguda odontogénica en adolescente: Reporte de caso. (2019). Rev. Clin. Periodoncia Implantol. Rehabil. Oral, 12, 90-92. https://doi.org/10.4067/S0719-01072019000200090

3. Subdural empyema and unilateral pansinusitis due to a tooth infection. (2015). World Health Organisation (HINARI), 2015(2015), 1-3. https://doi.org/10.1136/bcr-2014-207666

4. Sinusitis maxilar de origen Odontogénica. Diagnóstico y tratamiento quirúrgico. (2013). Int. J. Odontostomat, 7(3), 421-426. https://scielo.conicyt.cl/scielo.php?script=sci_arttext\&pid=SO718381X2013000300014 


\section{CIENCIA\&SALUD}

5. Martinez L, Albañil R. Documento de consenso sobre etiología, diagnóstico y tratamiento de la sinusitis. Pediatría, Atención Primaria. Julio de 2019;XV(59):203-18.

6. Rinosinusitis odontogénica: Abordaje actual y comunicación de un caso. (2017). An Orl Mex, 62(1), 55-64. https://www.medigraphic.com/pdfs/anaotomex/aom-2017/aom171g.pdf

7. Bischhoffshausen K, Teuber C. Diagnóstico y tratamiento de la sinusitis maxilar odontogénica. Rev. Otorrinolaringol. Cir. Cabeza Cuello. 2019;79:357-365.

8. Chelsea, K. (2014). Fifteen-Year-Old girl with severe obstructive lung disease, pansinusitis, and failure to thrive. Clinical Pediatrics, 53(10), 1016-1018. https://doi.org/10.1177/0009922814533417

9. Sinusitis maxilares odontogénicas. Revisión de la literatura y caso clínico. (2018). labor dental clínica, 19(2), 70-89. https://www.revistalabordentalclinica.com/wp-content/uploads/2019/02/ sinusitis-maxilaes-odontog\%C3\%A9nicas.pdf

10. Dermatitis artefacta presenting as recurrent periorbital cellulitis. (2016). The Journal of Pediatrics, 175(237), 237-237.e http://dx.doi.org/10.1016/j.jpeds.2016.05.003

11. Septic cavernous sinus thrombosis associated with orbital cellulitis: A report of 6 cases and review of literature. (2019). The American Society of Ophthalmic Plastic and Reconstructive Surgery, Inc, 35(3), 272-280. https://doi.org/10.1097/IOP.000000000000123

12. Fokkens WJ, Lund VJ, Hopkins C, Hellings PW, Kern R., Reltsma S., et al European Position Paper on Rhinosinusitis and Nasal Polyps 2020 Rhinology. 2020 Suppl. 29; 1 - 464

13. Early cavernous sinus thrombosis following unilateral pansinusitis in a child. (2015). World Health Organisation (HINARI), 1-4. https://doi.org/10.1136/bcr-2014-208441

14. Nontraumatic orbital subperiosteal hematoma in a case of bernard- soulier syndrome with bilateral pansinusitis. (2019). The American Society of Ophthalmic Plastic and Reconstructive Surgery, Inc., 35(6), e134-e135. https://doi.org/10.1097/IOP.0000000000001463

15. Care and outcomes of canadian children hospitalised with periorbital and orbital cellulitis: Protocol for a multicentre, retrospective cohort study. (2019). World Health Organisation (HINARI), 9(12), 1-7. https://doi.org/10.1136/bmjopen-2019-035206 